\title{
Plasminogen as a prognostic biomarker for HBV-related acute-on-chronic liver failure
}

\author{
Daxian Wu, ${ }^{1,2}$ Sainan Zhang, ${ }^{1}$ Zhongyang Xie, ${ }^{1}$ Ermei Chen, ${ }^{1}$ Qunfang Rao, ${ }^{1}$ Xiaoli Liu, ${ }^{1}$ Kaizhou Huang, Jing Yang, ${ }^{1}$ Lanlan Xiao, \\ Feiyang Ji, ${ }^{1}$ Zhengyi Jiang, ${ }^{1}$ Yalei Zhao, ${ }^{1}$ Xiaoxi Ouyang, ${ }^{1}$ Danhua Zhu, ${ }^{1}$ Xiahong Dai, ${ }^{3}$ Zhouhua Hou, ${ }^{4}$ Bingjie Liu, ${ }^{5}$ Binbin Deng, ${ }^{4}$ \\ Ning Zhou, ${ }^{6}$ Hainv Gao, ${ }^{3}$ Zeyu Sun, ${ }^{1}$ and Lanjuan Li $^{1}$ \\ ${ }^{1}$ State Key Laboratory for Diagnosis and Treatment of Infectious Diseases; National Clinical Research Center for Infectious Diseases; Collaborative Innovation Center for Diagnosis and Treatment of Infectious \\ Diseases; The First Affiliated Hospital, College of Medicine, Zhejiang University, Hangzhou, China. ²Department of Infectious Diseases, The First Affiliated Hospital of Nanchang University, Nanchang, China. \\ ${ }^{3}$ Department of Infectious Diseases, Shulan Hospital of Hangzhou, Hangzhou, China. ${ }^{4}$ Department of Infectious Diseases, Xiangya Hospital of Central South University, Changsha, China. ${ }^{5}$ Department of \\ Infectious Diseases, First Affiliated Hospital, College of Medicine, Nanhua University, Hengyang, China. ${ }^{6}$ Department of Infectious Diseases, Second Xiangya Hospital, Central South University, Changsha, China.
}

BACKGROUND. HBV-related acute-on-chronic liver failure (HBV-ACLF) is hallmarked by high short-term mortality rates, calling for accurate prognostic biomarkers for initial risk stratification.

\begin{abstract}
METHODS. Three tandem mass tag-labeled (TMT-labeled) quantitative proteomic studies were performed on 10 patients with HBV-related acute hepatic decompensation and on 20 patients with HBV-ACLF. Candidate biomarkers were preliminarily verified in a cross-sectional cohort $(n=144)$ and further confirmed in 2 prospective cohorts $(n=207$ and $n=148)$.
\end{abstract}

RESULTS. Plasminogen, a potential prognostic biomarker for HBV-ACLF, was identified by TMT quantitative proteomics and preliminarily verified in the cross-sectional cohort. Further validation with a prospective cohort $(n=207)$ showed that plasminogen levels at admission were significantly lower $(P<0.001)$ in HBV-ACLF nonsurvivors than in survivors. The cumulative survival duration of patients with high plasminogen levels was significantly longer $(P<0.001)$ than that of patients with low plasminogen levels. During hospitalization, plasminogen levels significantly decreased $(P=0.008)$ in the deterioration group but significantly increased $(P<0.001)$ in the improvement group. Additionally, plasminogen levels gradually increased in survivors but gradually decreased in nonsurvivors. The P5 score, a prognostic panel incorporating plasminogen levels, hepatic encephalopathy occurrence, age, international normalized ratio (INR), and total bilirubin, was significantly superior to the Child-Pugh, Model for End-stage Liver Disease (MELD), Chronic Liver Failure Consortium ACLF (CLIF-C ACLF), Chinese Group on the Study of Severe Hepatitis B (COSSH), and HINT (a prognostic score based on hepatic encephalopathy occurrence, INR, neutrophil count, and thyroid-stimulating hormone) scores (all $P<0.05$ ). The performances of the plasminogen level and P5 score were validated in a second multicenter, prospective cohort $(n=148)$.

CONCLUSIONS. Plasminogen is a promising prognostic biomarker for HBV-ACLF, and sequential plasminogen measurements could profile the clinical course of HBV-ACLF. P5 is a high-performance prognostic score for HBV-ACLF.

FUNDING. The National Key Research and Development Program (2017YFC1200204); the National Natural Science Foundation of China (81400589, 81600497); the Foundation for Innovative Research Groups of the National Natural Science Foundation of China (81121002); the Chinese High-Tech Research and Development Programs (2012AA020204); the National S\&T Major Project (2012ZX10002004); and the Zhejiang Provincial Medicine and Health Science and Technology Project (2016147735).

\section{Introduction}

Acute-on-chronic liver failure (ACLF) is a life-threatening clinical condition that occurs in patients with preexisting chronic liver disease (CLD). It can progress rapidly and has a mortality rate of more than $50 \%$, even after aggressive treatments $(1-3)$. HBV infection is a major etiology of ACLF, and there are currently 350 million indi-

Conflict of interest: The authors have declared that no conflict of interest exists. Copyright: ( 2020, American Society for Clinical Investigation.

Submitted: May 20, 2019; Accepted: January 14, 2020; Published: March 16, 2020. Reference information: J Clin Invest. 2020;130(4):2069-2080.

https://doi.org/10.1172/JCl130197. viduals infected with $\mathrm{HBV}$ worldwide (4). In most Asian countries, HBV-related ACLF (HBV-ACLF) accounts for over 70\% of ACLF cases $(5,6)$. Reports have shown that the patient's condition at admission is linked to the outcome of $\operatorname{ACLF}(1,7)$; thus, biomarkers that enable early and accurate risk stratification are needed. Additionally, ACLF is a highly dynamic process; therefore, sequential assessments of biomarkers that reflect the course of liver failure during hospitalization may enhance the management of patients with ACLF (7-9). However, to our knowledge, no biomarker has satisfactorily addressed these challenges.

Patients with CLD who experience an episode of acute hepatic insult causing liver dysfunction are considered to have acute 
hepatic decompensation (AHD), which can further progress to ACLF hallmarked by multiorgan failure and high mortality rates. Therefore, AHD is considered a pre-ACLF condition, and the prognoses for AHD and ACLF differ considerably $(2,10)$. The emergence of quantitative proteomics technologies has facilitated the high-throughput discovery of candidate biomarkers, particularly noninvasive blood biomarkers, of liver pathophysiology such as those for liver regeneration (11), hepatocyte differentiation and dedifferentiation (12), and acute injury and failure $(13,14)$. However, few studies have focused on biomarkers distinguishing ACLF from AHD, or ACLF survivors from nonsurvivors.

Here, we report the discovery of a promising prognostic biomarker for HBV-ACLF. First, we used isobaric labeling-based quantitative proteomics to assess ACLF-associated proteomic changes. Second, on the basis of the differentially expressed proteins (DEPs) identified in patients with ACLF, we verified several candidate biomarkers that allowed differentiation between ACLF nonsurvivors and survivors in a cross-sectional cohort. Third, we confirmed the prognostic power of plasminogen, the most promising biomarker, in a prospective, longitudinal cohort and developed a prognostic score (the P5 score) based on plasminogen levels, hepatic encephalopathy occurrence, age, international normalized ratio (INR), and total bilirubin. Finally, the prognostic value of plasminogen and the P5 score was further validated in a second multicenter, prospective cohort.

\section{Results}

Proteomics analysis. The overall design of this study is shown in Figure 1 . During the discovery stage, 729,699 , and 703 proteins were identified in tandem mass tag-labeled (TMT-labeled) proteomics experiments 1, 2, and 3, respectively (raw data are available at https://www.iprox.org/page/project.html?id=IPX0001802000; project ID: IPX0001802000). In total, 584 proteins were identified in all 3 experiments (Figure 2A). Using a FDR filter under 0.05 with a difference of 1.5 -fold or more, we identified 136 DEPs among patients with HBV-related AHD (HBV-AHD) and those with HBV-ACLF (Figure 2B). As expected, these patients could be distinguished in an unsupervised clustering analysis based on overall expression trends of DEPs (Figure 2C). Gene Ontology (GO) enrichment analyses revealed that the molecular functions of DEPs were primarily related to complement activity, protease inhibitor activity, and transporter activity, whereas the main biological processes involved protein metabolism, the immune response, and energy pathways. As for the cellular components term in the GO analysis, DEPs were mainly localized in extracellular components, exosomes, and lysosomes (Supplemental Figure 1, A-C; supplemental material available online with this article; https://doi. org/10.1172/JCI130197DS1). Hepatocyte nuclear factor-1 $\alpha$ (HNF$1 \alpha)$, followed by estrogen-related receptor $\alpha(\operatorname{ESRR} \alpha)$, and nuclear receptor subfamily 4 group A member 2 (NR4A2) were predicted to be upstream transcription factors related to DEPs in patients with HBV-ACLF (Figure 2D). Western blot analysis showed a gradually decreasing level of HNF-1 $\alpha$ in healthy controls (HCs) compared with patients with chronic hepatitis B (CHB), HBV-related liver cirrhosis (LC), HBV-AHD, or HBV-ACLF (Figure 2E). To validate the transcriptional connection between HNF- $1 \alpha$ and plasminogen, we observed a dose-dependent inhibition of plasminogen expres- sion in HepG2 cells treated with HNF-1 $\alpha$ siRNA (Supplemental Figure 2). Additionally, pathway enrichment analysis showed that DEPs were overrepresented in the complement and coagulation cascades, cholesterol metabolism, and Staphylococcus aureus infections (Figure 3A). Also, those DEPs enriched in the complement and coagulation cascades were downregulated in HBV-ACLF survivors compared with HBV-AHD patients and further downregulated in HBV-ACLF nonsurvivors (Figure 3, B-D).

Biomarkers predict the outcome for patients with $H B V-A C L F$. Of the 136 DEPs between the HBV-AHD and HBV-ACLF patients, 14 were downregulated and 2 were upregulated in HBV-ACLF nonsurvivors compared with those in survivors (Supplemental Table 1). Multivariate principal component analysis (PCA) showed that these candidate biomarkers clearly separated HBV-AHD patients, HBV-ACLF survivors, and HBV-ACLF nonsurvivors (Figure 4A). We built supervised orthogonal partial least squares discriminant analysis (OPLS-DA) models to identify protein signatures that could distinguish patients with HBV-ACLF from those with HBV-AHD, and HBV-ACLF nonsurvivors from survivors (Figure 4, B and C). Plasminogen, pigment epithelium-derived factor (SERPINF1), scavenger receptor cysteine-rich type 1 protein M130 (CD163), complement factor B (CFB), coagulation factor IX (F9), kininogen-1 (KNG1), and complement factor I (CFI) made the greatest contributions to differentiating HBV-ACLF survivors from nonsurvivors (predictive variable importance in projection [VIPpred] >1; Supplemental Table 1).

Preliminary validation of biomarkers. Among the 7 candidate biomarkers with a VIPpred greater than 1, the liver-specific proteins plasminogen, CFB, F9, KNG1, and CFI were validated in a cross-sectional cohort. As shown in Supplemental Figure 3, the levels of these 5 proteins decreased with increasing HBV disease severity (all $P<0.001$ ). Importantly, plasminogen and CFI levels in ACLF nonsurvivors were significantly lower than those in survivors $(P<0.05)$, and CFB and F9 levels tended to be lower in nonsurvivors (both $P<0.10$ ). The area under the receiver operating characteristics curves (AUCs) of plasminogen and CFI for the prediction of ACLF outcomes in the cross-sectional cohort were $0.770(0.610-0.888)$ and 0.687 (0.521-0.824), respectively.

Validation of plasminogen in a prospective, longitudinal $\mathrm{HBV}$ $A C L F$ cohort. Given that plasminogen was suggested to be the most promising prognostic biomarker in the preliminary validation (Supplemental Table 1 and Supplemental Figure 3), we next focused on the prognostic utility of plasminogen in a prospective HBV-ACLF cohort (cohort 2, $n=207$ ).

Of the patients with HBV-ACLF, $61.8 \%$ had prior cirrhosis, $78.2 \%$ were men, and $32.1 \%$ died within 30 days. The most frequent precipitating event for HBV-ACLF was HBV reactivation (60.9\%), followed by bacterial infection (36.7\%) and drug use (13.5\%).

The organs that failed most frequently, in order, were the liver (72.5\%), coagulation system (28.5\%), and lungs (24.6\%). Kidney and circulation failure were rare in patients with HBV-ACLF $(1.4 \%$ and $1.9 \%$, respectively). Of these patients, $44.0 \%$ had singleorgan failure, whereas $24.2 \%$ and $14.0 \%$ had 2 and 3 or more failed organs, respectively.

As shown in Figure 5A, the plasminogen level at admission in the nonsurvivor group was significantly lower than that in the survivor group (32.69 [29.39-39.89] vs. 48.57 [40.91-61.38] $\mu \mathrm{g} / \mathrm{mL}$, 


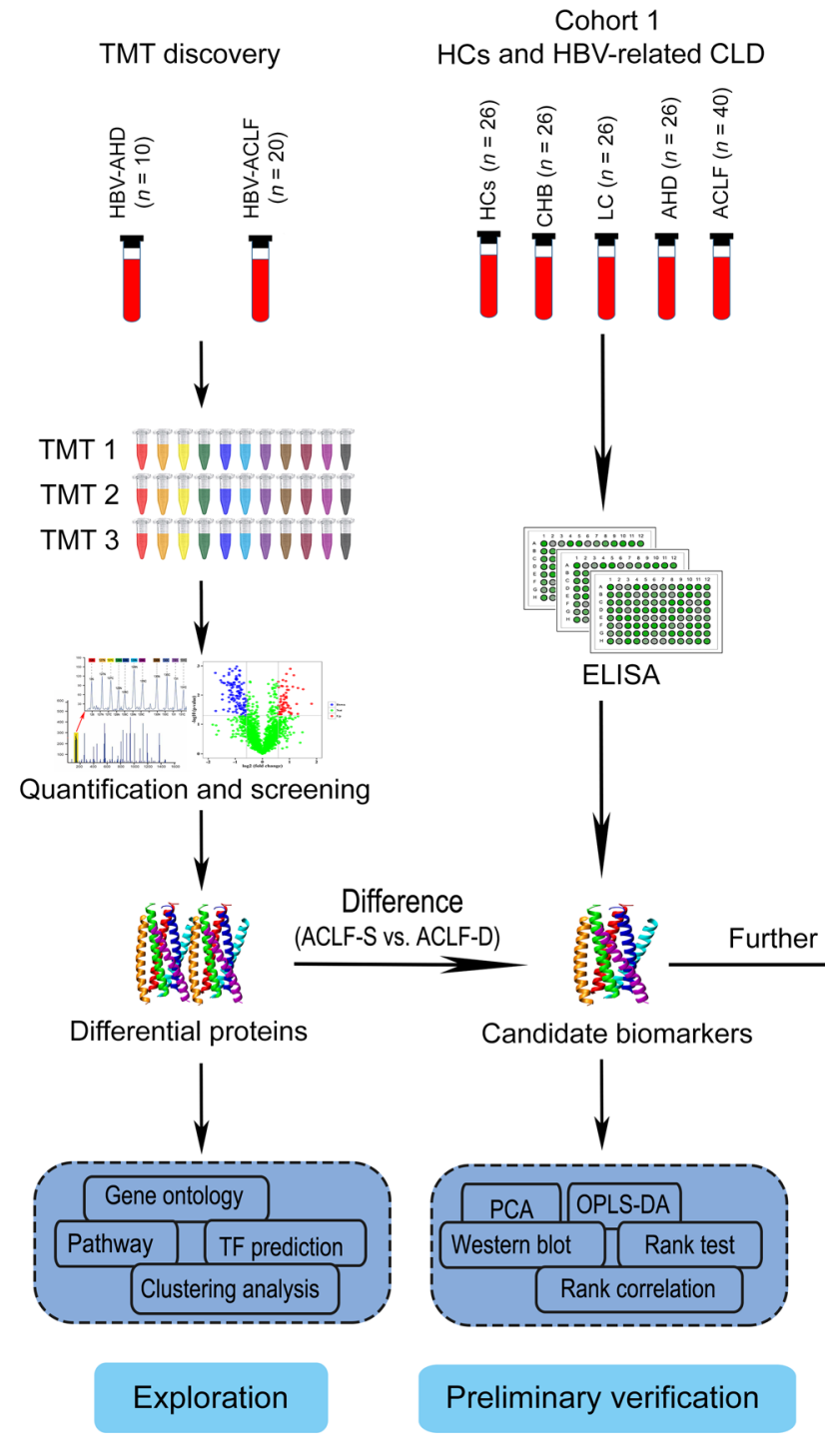

$\begin{array}{cc}\text { Cohort } 2 & \text { Cohort } 3 \\ \text { (derivation) } & \text { (validation) }\end{array}$

Cohort 4

(derivation) (validation) HCs and non-HBV-related CLD

s and HBV-related CLD
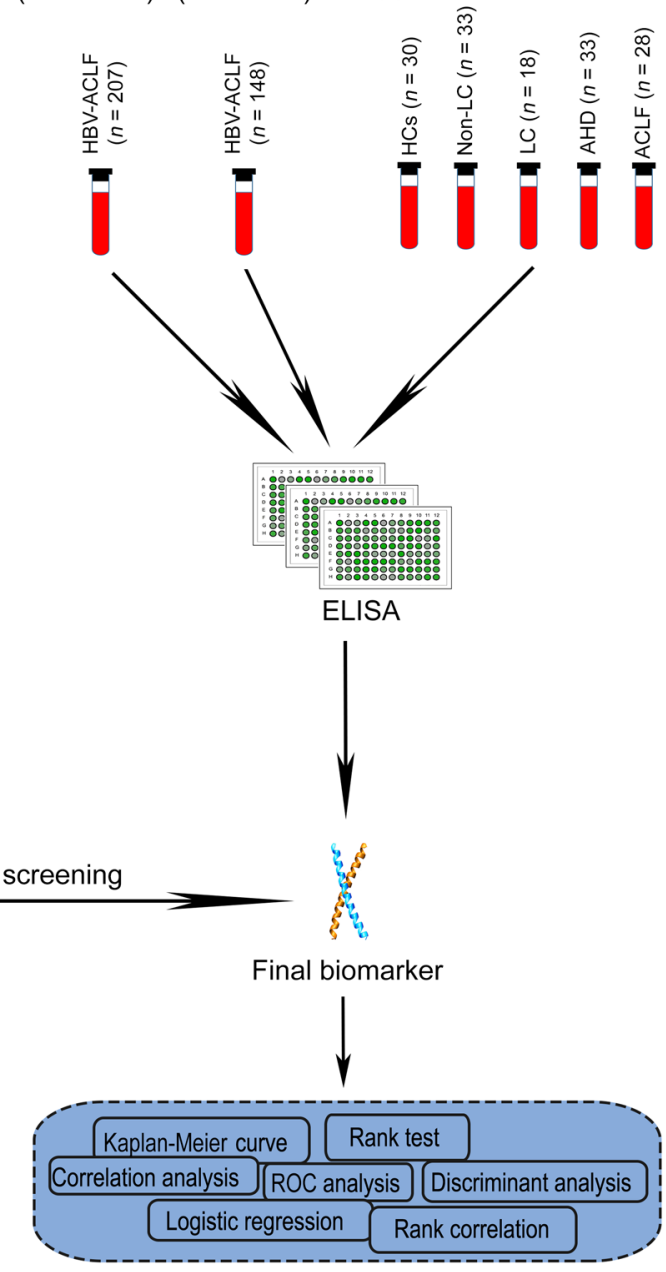

Further verification

Figure 1. Study design. First, HBV-ACLF DEPs and candidate prognostic biomarkers differentiating HBV-ACLF nonsurvivors from survivors were identified with TMT-labeled proteomics. Second, the candidate biomarkers were preliminarily validated with a cross-sectional cohort $(n=144)$ of HBV-related CLDs by ELISA. Third, a final promising prognostic biomarker was further screened from the candidate prognostic biomarkers and validated with 2 prospective HBV-ACLF cohorts $(n=207$ and $n=148)$ by ELISA. Finally, another cross-sectional cohort of individuals $(n=142)$ with autoimmune or alcoholic CLD were enrolled to determine whether the final prognostic biomarker could be extended to ACLF caused by non-HBV etiologies. ACLF-S, ACLF survivors; ACLF-D, ACLF deaths; TF, transcription factor.

$P<0.001)$. The AUC of plasminogen levels for 30-day mortality was 0.848 , with a sensitivity of 0.688 and a specificity of 0.893 at an optimal cut-off value of 35.47 (Figure 5B). We divided patients with ACLF into high-plasminogen $(>35.5 \mu \mathrm{g} / \mathrm{mL})$ and low-plasminogen $(\leq 35.5 \mu \mathrm{g} / \mathrm{mL})$ groups according to the optimal cut-off value. The cumulative survival rate of the high-plasminogen group was significantly higher than that of the low-plasminogen group $(P<0.0001$; Figure $5 C)$. The plasminogen level at admission decreased as the number of failed organs increased from 0 to more than 3 (52.03 [45.71-63.28], 50.74 [41.07-62.11], 39.90 [34.11-44.90], and 32.61 [25.42-35.30] $\mu \mathrm{g} / \mathrm{mL}, P<0.001$; Figure $5 \mathrm{~F})$. Additionally, the plasminogen levels at admission were markedly lower in patients with coagulation failure and cerebral failure than in those without these conditions (both $P<0.001$ ). The plas- minogen levels were slightly lower in patients with liver failure and respiratory failure than in those without these conditions $(P$ $=0.042$ and $P=0.049$, respectively). We observed no significant difference in plasminogen levels between patients with circulation failure and those without (Figure 5, G-K).

Sequential plasminogen measurements reflect the clinical course of HBV-ACLF. To observe the dynamic alterations of plasminogen during the patients' hospitalization, we assessed HBV-ACLF nonsurvivors with available 7-day follow-up serum samples ( $n$ = 36) and HBV-ACLF survivors with available 14-day follow-up serum samples $(n=48)$. We found that plasminogen levels gradually increased in survivors $(P<0.001)$ and gradually decreased in nonsurvivors $(P=0.019)$ (Figure $6 \mathrm{~A})$. Among these 84 patients, 18 experienced a deterioration in their condition, 30 had fluctua- 


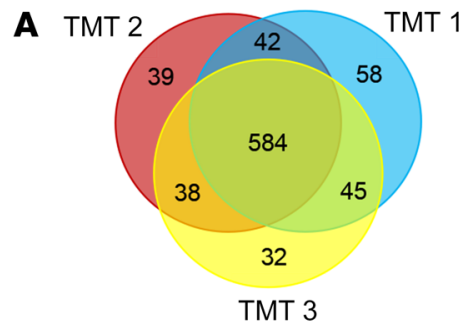

D

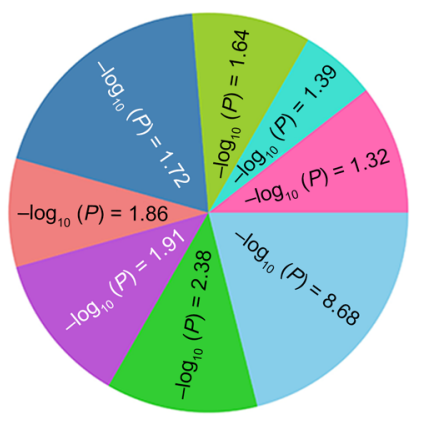

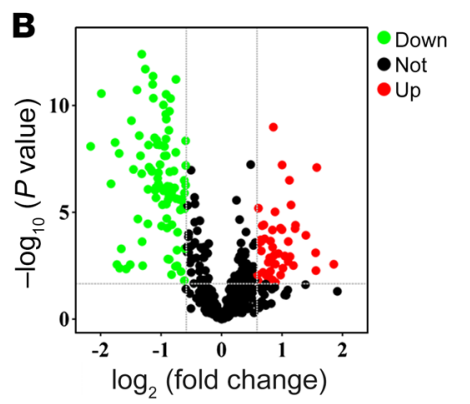

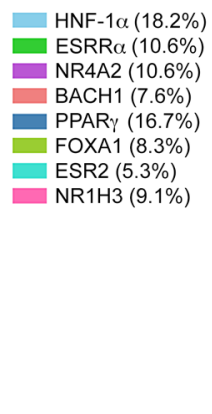

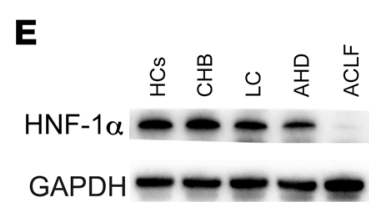

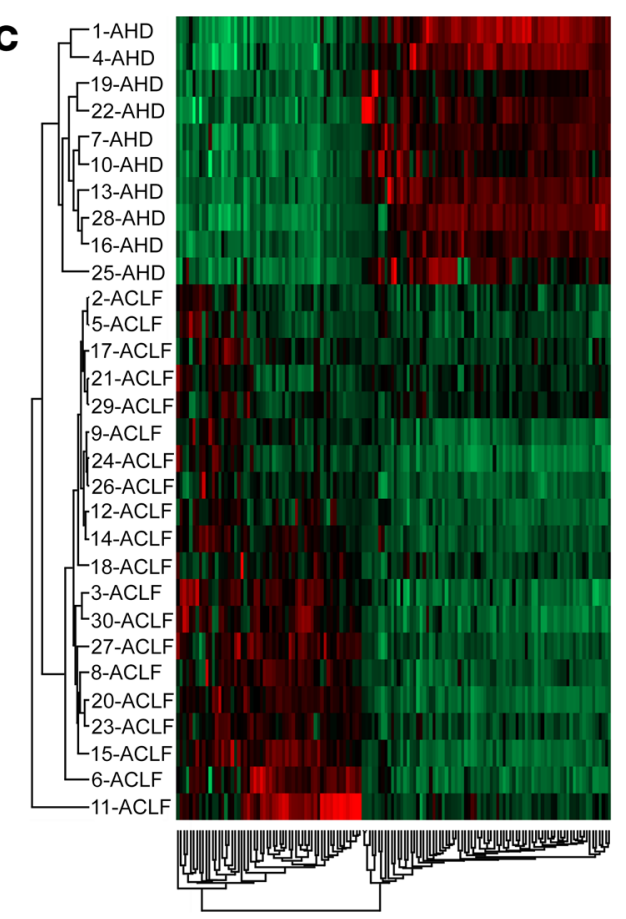

Figure 2. Bioinformatics analysis of proteomics data. (A) Venn diagram of the 3 TMT experiments. (B) Volcano plot of the 136 DEPs between AHD and ACLF patients. (C) Unsupervised clustering heatmap of the 136 DEPs between AHD and ACLF patients. Up, upregulated; Down, downregulated; Not, no change. (D) Prediction of upstream transcription factors related to DEPs. The value in parentheses indicates the percentage of proteins in the $136 \mathrm{DEPs}$ predicted to be regulated by each TF, and the value in each sector indicates hypergeometric $P$ value of the TF enrichment analysis. (E) Expression of HNF$1 \alpha$ in liver tissues. All patients with LC, AHD, or ACLF had an underlying HBV infection. BACH1, transcription regulator protein BACH1; PPAR $\gamma$, peroxisome proliferator-activated receptor $\gamma$; FOXA1, hepatocyte nuclear factor 3- $\alpha$; ESR2, estrogen receptor $\beta$; NR1H3, oxysterols receptor LXR- $\alpha$.

tions in their condition, and 36 experienced improvement in their condition during hospitalization (Supplemental Table 2). Compared with plasminogen levels at admission, the levels at the final follow-up assessment (14 days after admission or discharge) were significantly decreased in the deterioration group $(P=0.008)$ but significantly increased in the improvement group $(P<0.001)$, and were unchanged in the fluctuation group $(P=0.943)$ (Figure $6 \mathrm{~B})$. In summary, plasminogen levels closely correlated with the clinical course of HBV-ACLF.

Development and evaluation of a plasminogen-based prognostic model. We next evaluated the performance of plasminogen in combination with clinical parameters at admission to predict the outcomes of HBV-ACLF using the derivation cohort (cohort 2). Univariate and multivariate logistic regression analyses showed that plasminogen levels, age, hepatic encephalopathy (HE) occurrence, total bilirubin (TBil) levels, and the INR were independent prognostic factors for HBV-ACLF (Table 1). Therefore, we developed a prognostic panel (P5) based on the following 5 parameters: $\mathrm{P} 5=4.3 \times \ln ($ age $)+2.3 \times(\mathrm{HE})+2.1 \times \ln (\mathrm{TBil})+4.7 \times \ln (\mathrm{INR})-2.9$ $\times \ln$ (plasminogen), where $\mathrm{HE}=\mathrm{O}$ for patients without $\mathrm{HE} ; \mathrm{HE}=1$ for patients with mild $\mathrm{HE}$ (grades 1-2); and $\mathrm{HE}=2$ for patients with severe HE (grades 3-4). The P5 score for the nonsurvivor group was significantly higher than that for the survivor group (20.05 $[17.74,21.89]$ vs. 13.86 [12.05, 15.80], $P<0.0001$; Figure 7A). The P5 score was positively associated with the Child-Pugh score $(r=$ $0.54, P<0.001)$ and more strongly associated with the Model for End-stage Liver Disease (MELD), Chronic Liver Failure Consor- tium ACLF (CLIF-C ACLF), Chinese Group on the Study of Severe Hepatitis B (COSSH), and HINT (A prognostic score based on hepatic encephalopathy occurrence, INR, neutrophil count, and thyroid-stimulating hormone) scores $(r=0.78,0.80,0.88$, and 0.89 , respectively; all $P<0.001$; Supplemental Figure 4$)$. The AUC of the P5 score for 30 -day mortality was 0.955 , with a sensitivity of 0.896 and a specificity of 0.906 at an optimal cut-off value of 16.95 (Supplemental Table 3). The P5 score showed significantly greater predictive power for 30-day mortality for HBV-ACLF patients than did the Child-Pugh, MELD, CLIF-C ACLF, COSSH, and HINT scores (all $P<0.05$; Figure $7 \mathrm{~B}$ and Supplemental Tables 3 and 4 ). When patients were divided into risk subgroups according to the optimal P5 cut-off value, we found that the mortality rate of the high-risk (P5 $>17)$ group was significantly higher than that of the low-risk $(\mathrm{P} 5 \leq 17)$ group $(74.1 \%$ vs. $3.3 \%, P<0.001)$. The cumulative survival rate for the high-risk $\mathrm{P} 5$ group was significantly lower than that for the low-risk P5 group $(P<0.001$; Figure $7 C)$.

Validation of plasminogen and $P 5$ in an external, prospective cohort. We used an external, prospective HBV-ACLF cohort (cohort 3) from 3 hospitals to validate the performance of plasminogen levels and the P5 score. The comparisons of clinical and laboratory characteristics at admission between the derivation and validation cohorts are listed in Supplemental Table 5. Consistent with the results from the derivation cohort, plasminogen levels in the nonsurvivor group were significantly lower than those in the survivor group (33.60 [27.86-42.62] vs. 51.02 [40.40-65.58] $\mu \mathrm{g} / \mathrm{mL}, P<0.001$; Figure $5 \mathrm{~A}$ ). The plasminogen AUC for 30 -day 
A

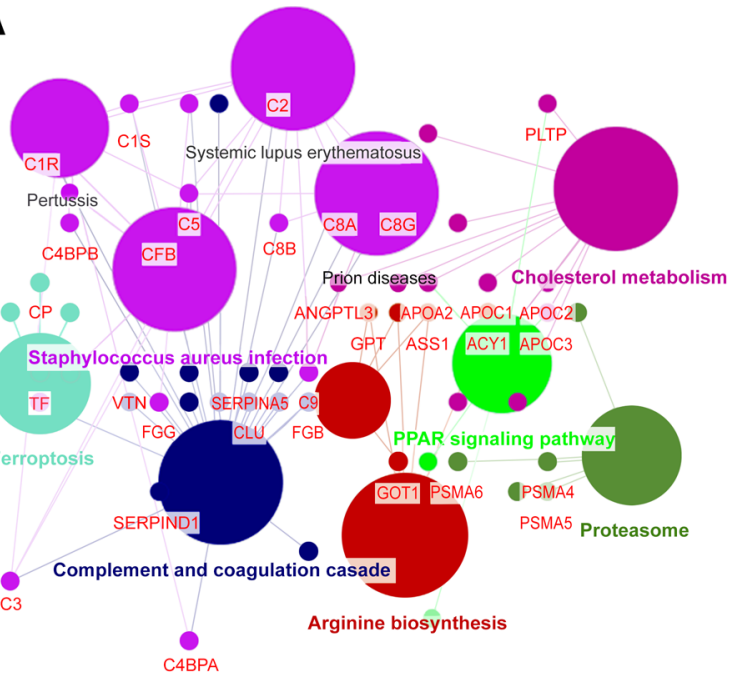

Figure 3. Enriched KEGG pathways. (A) Schematic representation of the 11 enriched Kyoto Encyclopedia of Genes and Genomes (KEGG) pathways (all $P<0.01$ ). Small dots indicate proteins, and large dots indicate enriched pathways. (B) Interactions of proteins associated with the complement and coagulation cascades. Blue indicates reference expression levels, and green indicates downregulation (magnitude is indicated by color intensity). All proteins shown were downregulated in HBV-ACLF survivors and to a greater degree in HBV-ACLF nonsurvivors compared with patients with HBV-AHD. (C and D) Relative expression levels of DEPs involved in the complement and coagulation cascades. Rank correlation was analyzed by the Spearman method. ${ }^{*} P<0.05$ and ${ }^{* * *} P$ $<0.001$. C1R, complement C1r subcomponent; C1S, complement C1S subcomponent; $C 2$, complement $\mathrm{C} 2 ; \mathrm{C}$, complement $\mathrm{C} 3$; $C 4 \mathrm{BPA}, \mathrm{C} 4 \mathrm{~b}$-binding protein $\alpha$ chain; $\mathrm{C} 4 \mathrm{BPB}$, C4b-binding protein $\beta$ chain; $C 5$, complement $C 5$; C6, complement C6; C8A, complement component C8 $\alpha$ chain; C8B, complement component C8 $\beta$ chain; C8C, complement component $\mathrm{C8} \gamma$ chain; $\mathrm{C9}$, complement component C9; CFB, complement factor B; CFI, complement factor I, CLU, clusterin; CPB2, carboxypeptidase B2; F2, prothrombin; F5, coagulation factor V; F9, coagulation factor IX; FGA, fibrinogen $\alpha$ chain; FGB, fibrinogen beta chain; FGG, fibrinogen $\gamma$ chain; KNG1, kininogen-1; PLG, plasminogen; PROC, vitamin K-dependent protein C; SERPINA5, plasma serine protease inhibitor; SERPINC1, antithrombin-III; SERPIND1, heparin cofactor 2; VTN, vitronectin.

B
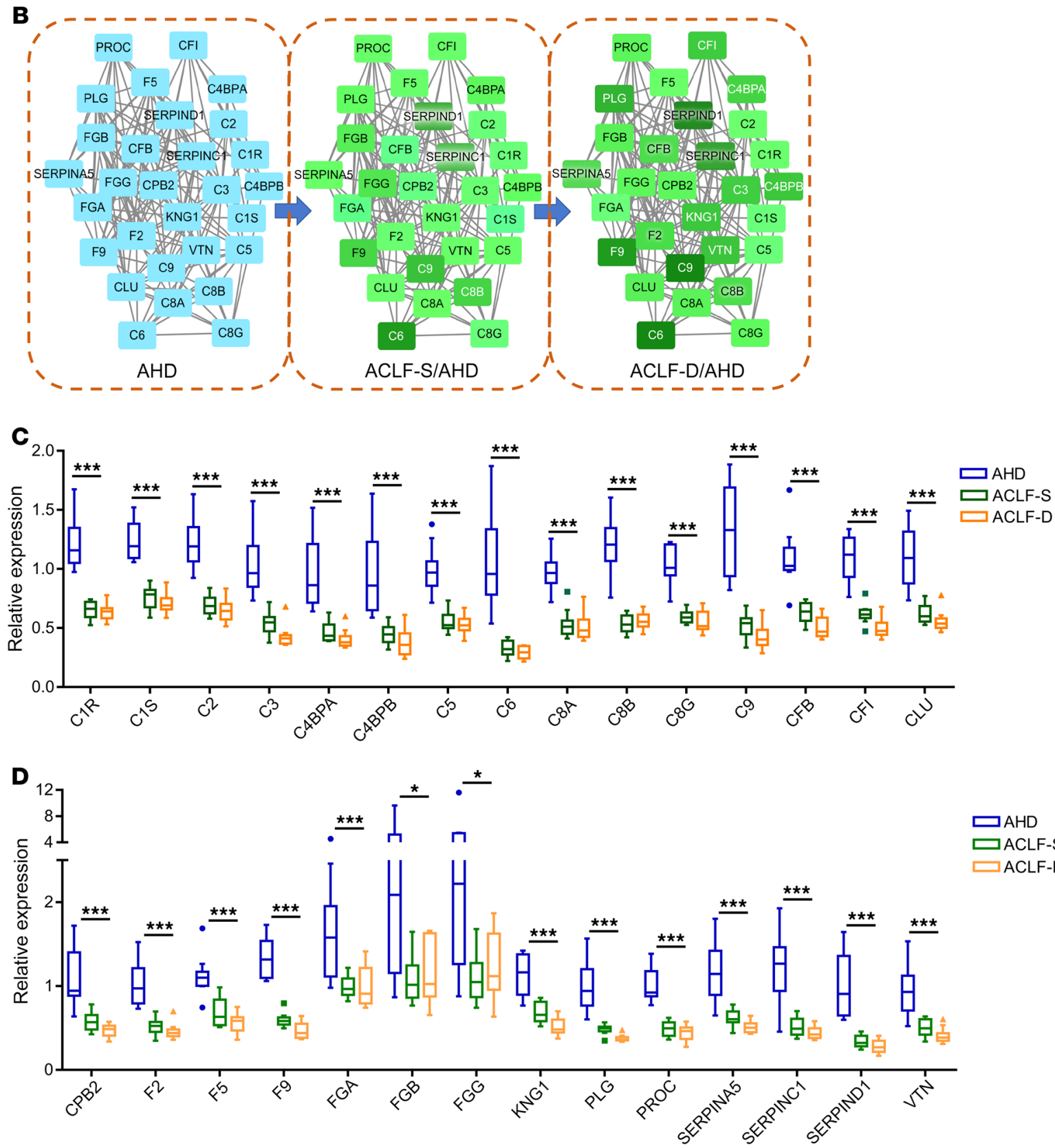

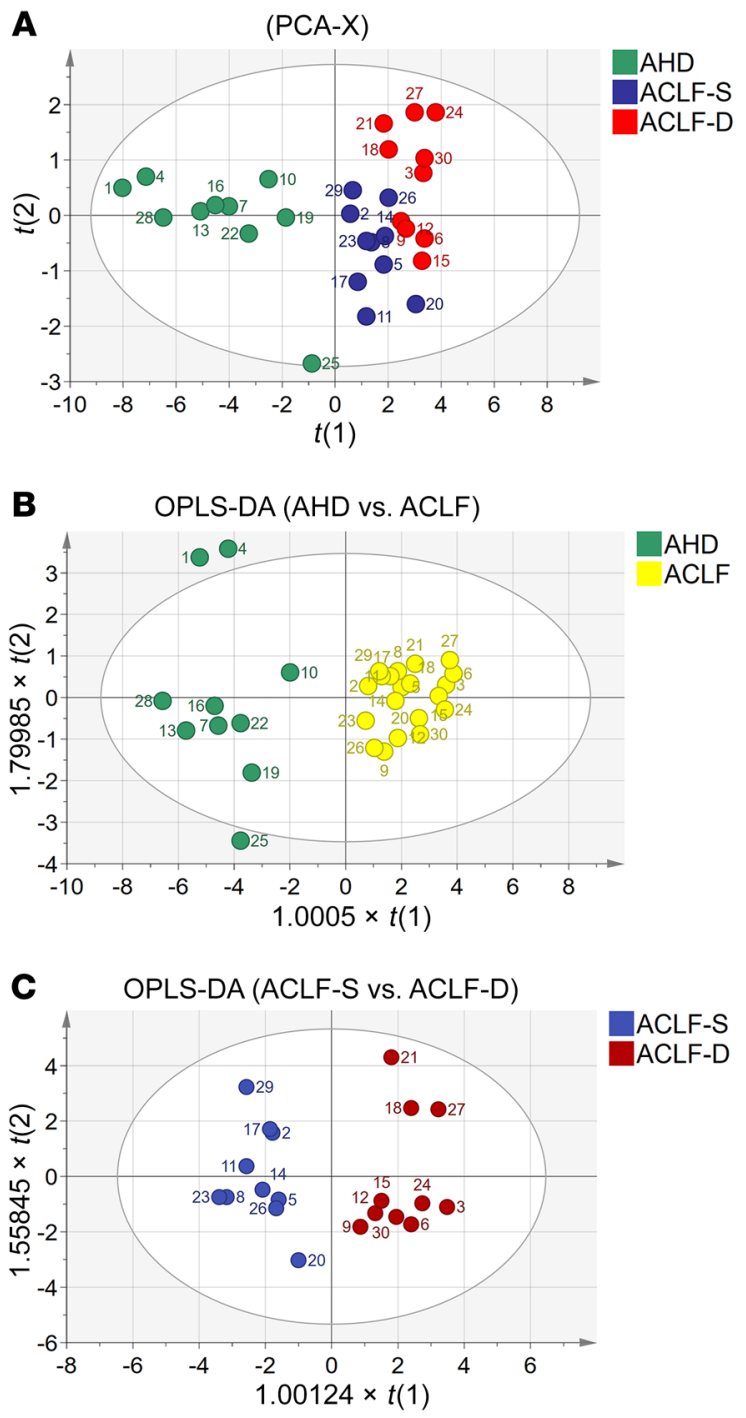

mortality was 0.812 , with a sensitivity of 0.762 and a specificity of 0.717 (Figure 5D). The cumulative survival duration for patients with high plasminogen levels was significantly longer than that for patients with low plasminogen levels $(P<0.0001$; Figure $5 \mathrm{E})$. Plasminogen levels decreased as the number of failed organs increased $(P<0.001$; Figure $5 F)$. Plasminogen levels at admission were dramatically lower in patients with coagulation failure and cerebral failure than in those who did not have these conditions (both $P<0.001$ ). We observed no significant difference in plasminogen levels between patients with liver failure, respiratory failure, or circulation failure and those without (Figure 5, G-K). Thus, the relationships between plasminogen levels and the number and type of failed organs were similar to those in the derivation cohort.

Nonsurvivors had a significantly higher P5 score than did survivors $(19.69$ [16.91, 22.39] vs. 13.80 [12.45, 15.92], $P<0.0001$; Figure 7A). As in the derivation cohort, the $\mathrm{P} 5$ score was positively associated with the Child-Pugh and MELD scores $(r=0.48$ and 0.60 , respectively; $P<0.001$ ) and more strongly associated with the CLIF-C ACLF, COSSH, and HINT scores $(r=0.77,0.88$, and 0.88 , respectively; all $P<0.001$; Supplemental Figure 5). The AUC of the P5 score for 30-day mortality was 0.907 , which was
Figure 4. PCA and OPLS-DA of candidate biomarkers. (A) Unsupervised PCA model of patients with AHD, ACLF-S, or ACLF-D showing a clear separation between them. OPLS-DA models differentiating (B) AHD from ACLF and (C) ACLF-S from ACLF-D. All patients with AHD or ACLF had an underlying HBV infection.

significantly higher than that of the Child-Pugh, MELD, and HINT scores (AUC $=0.718,0.680$, and 0.856 respectively, all $P<$ 0.05 ) and tended to be higher than those of the CLIF-C ACLF and COSSH scores (AUC $=0.857$ and $0.868 ; P=0.0566$ and 0.0686; respectively; Figure 7D and Supplemental Table 4). The AUCs are listed in Supplemental Table 3. The mortality rate for the high-risk P5 group (P5 $>17)$ was significantly higher than that for the lowrisk $\mathrm{P} 5$ group ( $\mathrm{P} 5 \leq 17)(72.1 \%$ vs. $10.5 \%, P<0.001$, Figure $7 \mathrm{E})$.

Estimation of HBV-ACLF mortality based on the P5 score. To facilitate the clinical application of the P5 score, we estimated the HBV-ACLF mortality rates in the derivation and validation cohorts on the basis of the P5 score using Fisher's canonical discriminant analysis. The mortality rate approached zero when the P5 score was lower than 10; increased to $18.3 \%$ and $90.5 \%$ for $\mathrm{P} 5$ scores of 15 and 20, respectively; and reached $100 \%$ for P5 scores above 24 (Figure $7 F)$. For P5 scores from 15 to 20, the mortality rate of HBV-ACLF increased by $18.5 \%$ for each 1-point increment in the P5 score.

Performance of the P5 score in different HBV-ACLF subtypes. Among the patients with HBV-ACLF in the derivation and validation cohorts, 133 (37.5\%) did not have prior cirrhosis (noncirrhotic ACLF, type A), 137 (38.6\%) had prior compensated cirrhosis (compensated cirrhotic ACLF, type B), and 85 (23.9\%) had prior decompensated cirrhosis (decompensated cirrhotic ACLF, type C). For the prediction of outcomes for patients with type A HBV-ACLF, the AUC of the P5 score was significantly higher than that of the Child-Pugh, MELD, CLIF-C ACLF, and HINT scores (0.955 vs. $0.807,0.818,0.906$, and 0.921 respectively; all $P<0.05$ ). In addition, for the prediction of the outcomes for patients with type-B HBV-ACLF, the AUC of the P5 score was significantly higher than the Child-Pugh, MELD, CLIF-C ACLF, and COSSH scores (0.938 vs. $0.697,0.785,0.872$, and 0.874 , respectively; all $P<0.05$ ) and tended to be higher than the AUC of the HINT score (0.938 vs. $0.903, P=0.09)$. Finally, for the prediction of the outcomes for patients with type C HBV-ACLF, the P5 score was comparable to the other 5 scores ( 0.869 vs. $0.759,0.774,0.803,0.842$, and 0.791 , respectively; all $P>0.05$ ). The comparison between the P5 score and other scores according to HBV-ACLF subtype is shown in Supplemental Figure 6 and Supplemental Tables 6 and 7.

Plasminogen levels in autoimmune and alcoholic ACLF. In order to investigate whether the predictive potential of plasminogen could be extended to ACLF caused by non-HBV etiologies, the present study enrolled another cross-sectional cohort (cohort 4) that included $30 \mathrm{HCs}$ and 112 patients with CLD whose diseases were either autoimmune in origin or caused by alcohol (Supplemental Table 8). Surprisingly, plasminogen levels also gradually decreased from HCs to non-LC, to LC, to AHD, and then to ACLF. Plasminogen levels in ACLF nonsurvivors were significantly lower than those in survivors $(P<0.01$, Supplemental Figure 8). These encouraging results suggest that plasminogen may also serve as a prognostic biomarker for ACLF of other etiologies and warrant further studies. 
A

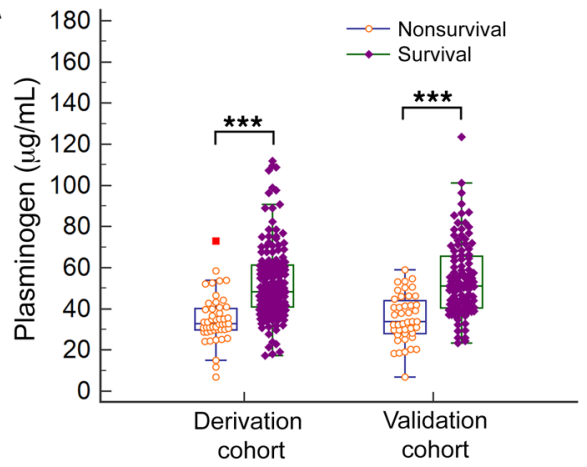

B

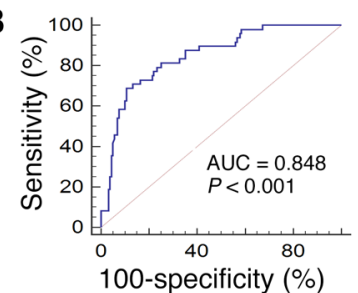

D

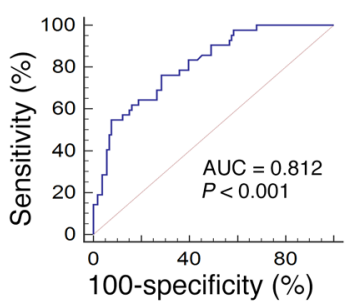

C

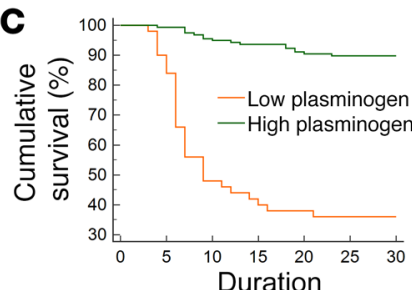

E

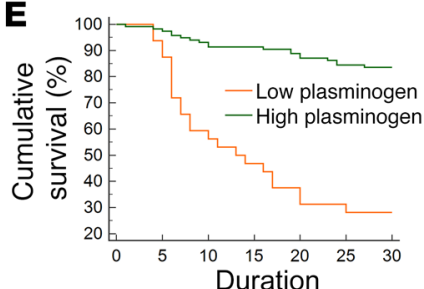

H

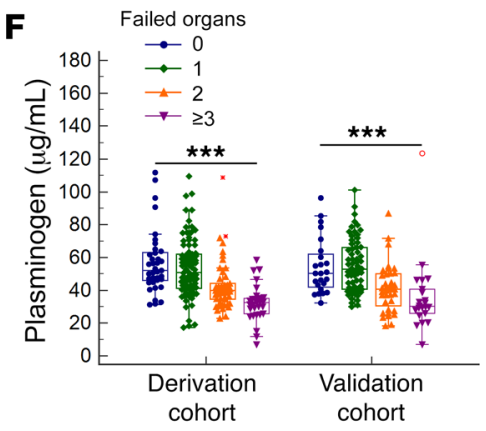

G

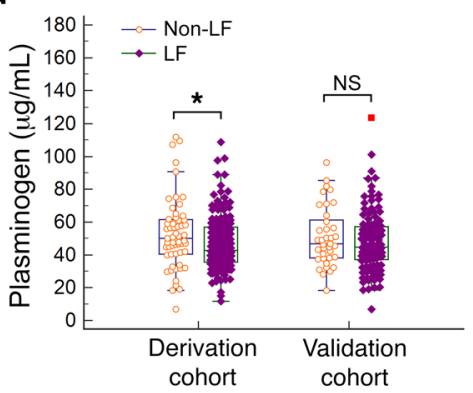

J

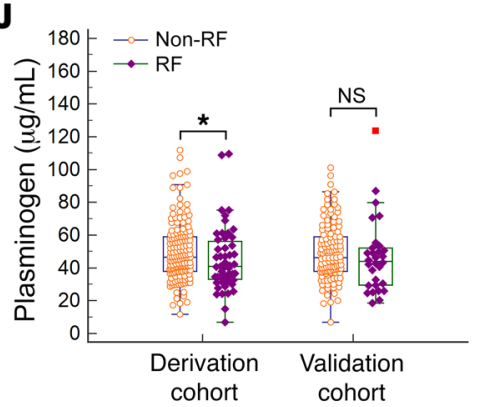

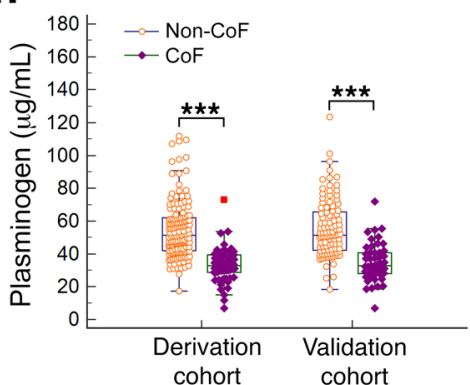

$\mathbf{K}$

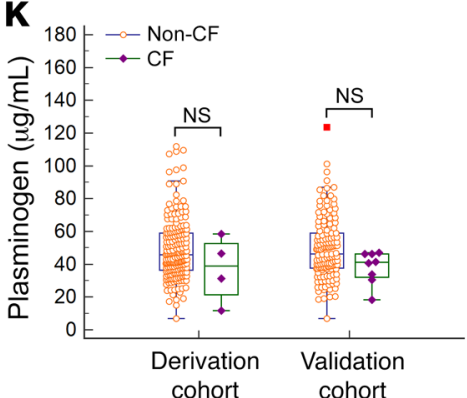

Figure 5. Association of plasminogen level at admission with HBV-ACLF prognosis and organ failure. (A) Plasminogen distribution for patients with HBV-ACLF in both the derivation and validation cohorts. Prediction of 30-day mortality according to plasminogen levels in patients with HBV-ACLF in the (B) derivation and (D) validation cohorts. Survival rates after 30 days of patients in the high- and low-plasminogen groups in the (C) derivation and (E) validation cohorts. Plasminogen levels at admission decreased with (F) increasing numbers of failed organs. Plasminogen distribution of HBV-ACLF patients with and without (G) liver failure (LF), (H) coagulation failure (CoF), (I) cerebral failure (CeF), (J) respiratory failure (RF), and (K) circulation failure (CF) in the derivation and validation cohorts. Plasminogen levels were compared using the Mann-Whitney $U$ test, rank correlation was analyzed by the Spearman method, and Kaplan-Meier plots were compared by log-rank test. ${ }^{*} P<0.05$ and ${ }^{* * *} P<0.001$.

\section{Discussion}

A few studies utilized proteomic approaches to identify biomarkers for HBV-ACLF (15-17). However, these studies were conducted with small sample sizes, and, consequently, the results were inconclusive. Additionally, these studies did not include HBV-AHD, which is difficult to differentiate from HBV-ACLF, did not attempt to differentiate HBV-ACLF survivors from nonsurvivors, and did not analyze the clinical course of HBV-ACLF in a prospective, longitudinal manner. In summary, the molecular signature of HBV-ACLF was still incomplete. Therefore, we performed this study with the aim of identifying effective biomarkers that reflect the progression of HBV-ACLF.

Unsupervised clustering of proteomics data showed that 136 DEPs could distinguish HBV-ACLF from HBV-AHD unambigu- ously, suggesting that these proteins might predict the occurrence of ACLF. Additionally, HNF-1 $\alpha$ was predicted to be the key transcriptional regulator that leads to significant alterations in DEPs during HBV-ACLF (Figure 2D). Moreover, hepatic expression of HNF-1 $\alpha$ was confirmed to gradually decrease from HCs to HBVACLF. HNF-1 $\alpha$ has been reported to regulate complex networks of biological pathways, including complement activation, the clotting cascade, and cholesterol metabolism (18-21). Coincidentally, these pathways were found to be enriched in the present study (Figure $3 \mathrm{~A})$. In addition, another study has revealed that 2 transcriptional activation sites of plasminogen are recognized by HNF-1 (22). The present study further revealed that expression of the plasminogen gene was decreased in HNF-1 $\alpha$-knockdown HepG2 cells and that 


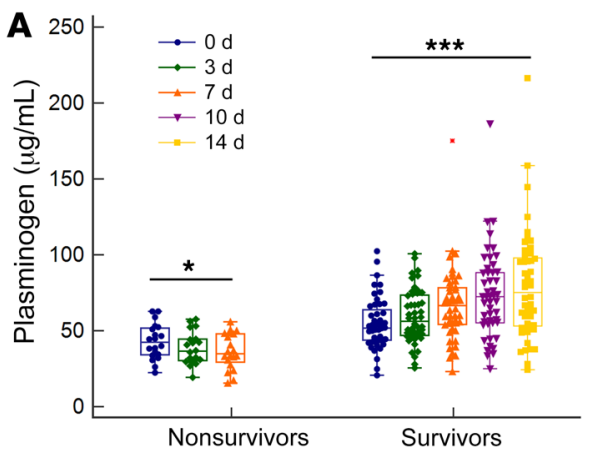

Nonsurvivors

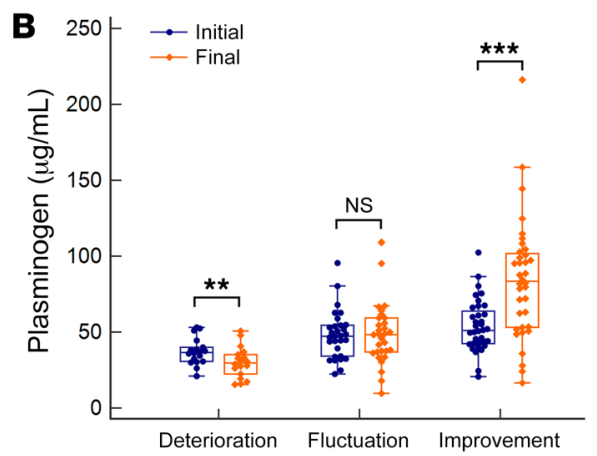

Figure 6. Longitudinal changes in plasminogen levels according to clinical course. (A) Longitudinal changes of plasminogen levels in HBV-ACLF survivors and nonsurvivors during hospitalization. (B) Changes in plasminogen levels between the initial (admission) and final (14 days after admission or discharge) assessments. Paired and repeated-measures data were analyzed by the Wilcoxon test and the Greenhouse-Geisser method, respectively. ${ }^{*} P<0.05,{ }^{* *} P<0.01$, and ${ }^{* *} P<0.001$. plasminogen levels were negatively correlated with the degree of knockdown. Thus, we postulate that decreased HNF-1 $\alpha$ levels lead to a deficiency in complement/clotting components and cholesterol metabolism during HBV-ACLF pathogenesis, but this concept requires further investigation.

When AHD progresses to ACLF, risk stratification is necessary to identify patients who are in urgent need of a liver transplant and those who are not (23). Indicators for this purpose, including soluble CD163 (24), macrophage inflammatory protein $3 \alpha$ (25), and connexin 43 (26), have been proposed. However, these indicators are still a long way from clinical application. Thus, we further refined biomarkers to differentiate HBV-ACLF nonsurvivors from survivors. Among the 16 candidate biomarkers, plasminogen, the most promising biomarker discovered by OPLS-DA, was verified in 2 prospective cohorts. We observed that a decreased plasminogen level at admission was markedly associated with mortality in patients with HBV-ACLF. During hospitalization, a 14-day longitudinal observation showed that plasminogen levels gradually increased in survivors, but the levels gradually decreased in nonsurvivors. These changes in plasminogen levels reflected the course of improvement, deterioration, and fluctuation. Notably, plasminogen levels were negatively associated with the number of failed organs and were markedly lower in patients with HBVACLF with cerebral failure or coagulation failure. These results robustly demonstrated that plasminogen is a promising biomarker for HBV-ACLF and that sequential plasminogen measurements reflect the clinical course of HBV-ACLF. Moreover, when analyzed together with the clinical parameters, plasminogen remained an independent prognostic factor for HBV-ACLF in a multivariate logistic regression analysis.

It would be expected that plasminogen, as a liver-specific protein, along with other coagulation factors, would dramatically decrease when liver functions are severely impaired. In theory, a decrease in plasminogen levels should parallel an increase in the INR value in patients with ACLF as a result of impaired synthetic functions of the liver. In this study, however, the plasminogen level was weakly correlated with the INR value $(r=0.55-0.58$, Supplemental Figure 7$)$, implying that plasminogen may be involved in additional pathological processes apart from the coagulation cascade during the pathogenesis of ACLF. In patients with liver failure, liver regeneration was commonly accompanied by necrosis and shown to be necessary for the patients' recovery. Plasminogen activation by the plasmin/ $\alpha 2$-antiplasmin system reportedly plays an important role in liv- er regeneration after injury (27). Therefore, markedly decreased plasminogen levels in patients with ACLF would hamper liver regeneration by reducing the production of hepatocyte growth factor and impeding remodeling $(28,29)$. Thus, patients with ACLF are subjected to a vicious circle of liver injury, plasminogen depletion, and impaired hepatocyte regeneration. Further investigations are needed to ascertain the mechanism underlying decreased plasminogen levels in patients with ACLF.

Because ACLF involves multiple systems and has a complex pathology $(1,9)$, a comprehensive prognostic score that includes indicators of multiple pathological processes is needed to predict the outcome of ACLF. Similar to previous reports (30-32), we demonstrated comparable abilities of CLIF-C ACLF, COSSH, and HINT scores to determine HBV-ACLF prognosis. Here, we showed that the P5 score, based on plasminogen levels and 4 other clinical parameters, outperformed the Child-Pugh, MELD, CLIF-C ACLF, COSSH, and HINT scores in both a single-center derivation cohort and a multicenter validation cohort. According to the estimated HBV-ACLF mortality rate, P5 scores below 15 and above 20 indicated good and poor prognoses, respectively, enabling patient stratification. In addition, $40.8 \%$ of patients with HBV-ACLF had P5 scores of 15 to 20, and the mortality rate for patients with HBVACLF increased by $18.5 \%$ for each 1-point increment in the P5 score in this range, facilitating a dynamic monitoring of the disease during hospitalization. Notably, the P5 score showed a considerably higher predictive power for HBV-ACLF types A and B, but its ability to predict type $C$ was comparable to that of the other scores. The latter may be due to the small sample size used. Therefore, the P5 score enables the prediction of outcomes for patients with ACLF and decision-making regarding the allocation of intensive care resources and priorities for urgent liver transplantation.

This study has several limitations. First, we identified 136 DEPs that could predict the occurrence of ACLF, which may provide a rich biomarker pool for HBV-ACLF diagnosis, but further refinement of the diagnostic biomarkers from this pool is needed. Second, we focused on plasminogen as a prognostic biomarker for HBV-ACLF and verified its performance in multiple cohorts; but whether the addition of other candidate biomarkers could increase the predictive power for HBV-ACLF has not been investigated. Third, whether plasminogen is a prognostic predictor of ACLF caused by other etiologies still needs further study, although the plasminogen levels were verified as having gradually decreased with increasing disease severity in a small cross-sectional cohort of individuals with autoimmune or alcoholic CLD. 


\section{Table 1. Characteristics at admission according to outcomes for the derivation cohort}

\begin{tabular}{|c|c|c|c|c|c|c|c|}
\hline \multirow[t]{2}{*}{ Variable } & \multirow[t]{2}{*}{ Total $(n=207)$} & \multirow[t]{2}{*}{ Nonsurvivors $(n=48)$} & \multirow[t]{2}{*}{ Survivors $(n=159)$} & \multicolumn{2}{|c|}{ Univariate logistic regression } & \multicolumn{2}{|c|}{ Multivariate logistic regression } \\
\hline & & & & $\mathrm{HR}(95 \% \mathrm{Cl})$ & $P$ & $\mathrm{HR}(95 \% \mathrm{Cl})$ & $P$ \\
\hline \multicolumn{8}{|c|}{ Clinical and laboratory parameters } \\
\hline Age (yr) & $48.26 \pm 12.47$ & $52.42 \pm 12.29$ & $47.00 \pm 12.28$ & $1.036(1.009-1.064)$ & 0.009 & $1.089(1.035-1.145)$ & 0.001 \\
\hline Fatigue & $10(4.8 \%)$ & $3(6.3 \%)$ & $7(4.4 \%)$ & $1.448(0.360-5.828)$ & 0.603 & & \\
\hline Alcoholism & $17(8.2 \%)$ & $5(10.4 \%)$ & $12(7.5 \%)$ & $1.125(0.648-1.955)$ & 0.675 & & \\
\hline HBV reactivation & $126(60.9 \%)$ & $34(70.8 \%)$ & $92(57.9 \%)$ & $1.769(0.881-3.553)$ & 0.109 & & \\
\hline Bacterial infection & $76(36.7 \%)$ & $25(52.1 \%)$ & $51(32.1 \%)$ & $2.302(1.193-3.257)$ & 0.013 & & \\
\hline Ascites (mild/severe) & $71 / 50$ & $16 / 13$ & $55 / 37$ & $1.106(0.737-1.660)$ & 0.625 & & \\
\hline UGIB & $29(14.0 \%)$ & $7(14.6 \%)$ & $22(13.8 \%)$ & $0.941(0.375-2.359)$ & 0.896 & & \\
\hline Cirrhosis & $128(61.8 \%)$ & $25(52.1 \%)$ & $103(64.8 \%)$ & $0.591(0.308-1.136)$ & 0.114 & & \\
\hline Decompensation history & $52(25.1 \%)$ & $8(16.7 \%)$ & $44(27.7 \%)$ & $0.523(0.227-1.205)$ & 0.128 & & \\
\hline $\mathrm{HE}$ (mild/severe) & $13 / 21$ & $6 / 19$ & $7 / 2$ & $11.163(4.952-25.163)$ & $<0.001$ & $9.472(3.154-28.448)$ & $<0.001$ \\
\hline Neutrophils $\left(\times 10^{9} / \mathrm{L}\right)$ & $4.30(3.10-6.30)$ & $5.95(3.95-8.38)$ & $4.00(3.00-5.70)$ & $1.214(1.089-1.354)$ & $<0.001$ & & \\
\hline $\mathrm{RBC}\left(\times 10^{12} / \mathrm{L}\right)$ & $4.03 \pm 0.75$ & $4.07 \pm 0.75$ & $4.01 \pm 0.75$ & $1.113(0.718-1.726)$ & 0.632 & & \\
\hline Platelets $\left(\times 10^{9} / \mathrm{L}\right)$ & $108.29 \pm 53.47$ & $104.50 \pm 44.98$ & $109.43 \pm 55.86$ & 0.998 (0. 992-1.004) & 0.575 & & \\
\hline Total protein (g/L) & $58.80 \pm 8.41$ & $58.57 \pm 7.47$ & $58.87 \pm 8.69$ & $0.971(0.913-1.034)$ & 0.360 & & \\
\hline Albumin (g/L) & $31.48 \pm 4.46$ & $31.24 \pm 4.50$ & $31.55 \pm 4.46$ & $0.996(0.958-1.035)$ & 0.669 & & \\
\hline $\operatorname{ALT}(\mathrm{U} / \mathrm{L})$ & $246.00(90.00-630.00)$ & $323.00(141.50-582.75)$ & $215.00(86.00-674.00)$ & $1.000(1.000-1.001)$ & 0.612 & & \\
\hline AST (U/L) & $184.00(91.00-386.00)$ & $265.50(132.75-507.75)$ & $157.00(86.00-349.00)$ & $1.001(1.000-1.002)$ & 0.023 & & \\
\hline Serum bilirubin (mg/dL) & $16.99(11.58-23.22)$ & $20.79(16.95-26.26)$ & $15.62(10.58-22.71)$ & $1.091(1.045-1.141)$ & $<0.001$ & $1.099(1.016-1.189)$ & 0.018 \\
\hline Creatinine (mg/dL) & $0.72(0.63-0.83)$ & $0.75(0.65-0.97)$ & $0.71(0.62-0.81)$ & $6.822(2.215-21.013)$ & 0.001 & & \\
\hline Potassium (mmol/L) & $4.19 \pm 0.60$ & $4.32 \pm 0.59$ & $4.15 \pm 0.60$ & $1.584(0.938-2.673)$ & 0.085 & & \\
\hline Sodium (mmol/L) & $137.61 \pm 3.88$ & $137.90 \pm 3.75$ & $137.52 \pm 3.92$ & $1.026(0.942-1.117)$ & 0.558 & & \\
\hline Cerebral, $n(\%)$ & $21(10.1 \%)$ & $19(39.6 \%)$ & $2(1.3 \%)$ & $3.940(2.734-23.125)$ & 0.001 & & \\
\hline Lung, $n(\%)$ & $51(24.6 \%)$ & $18(37.5 \%)$ & $33(20.8 \%)$ & $2.291(1.139-4.608)$ & 0.020 & & \\
\hline Circulation, $n(\%)$ & $4(1.9 \%)$ & $4(8.3 \%)$ & $0(0 \%)$ & $22.488(22.162-22.837)$ & 0.001 & & \\
\hline Kidney, $n(\%)$ & $3(1.4 \%)$ & $3(6.3 \%)$ & $0(0 \%)$ & $22.465(22.142-22.848)$ & 0.001 & & \\
\hline $\begin{array}{l}\text { Proportion of failed } \\
\text { organs }(1 / 2 / \geq 3)\end{array}$ & $91 / 50 / 29$ & $4 / 19 / 25$ & $87 / 31 / 4$ & 12.209 (5.995-24.868) & $<0.001$ & & \\
\hline Number of failed organs & $1.00(1.00-2.00)$ & $3.00(2.00-3.00)$ & $1.00(1.00-1.00)$ & $10.594(5.411-22.175)$ & $<0.001$ & & \\
\hline Child-Pugh & $10.71 \pm 1.55$ & $11.79 \pm 1.20$ & $10.38 \pm 1.50$ & $2.061(1.560-2.724)$ & $<0.001$ & & \\
\hline MELD & $22.50 \pm 5.38$ & $28.31 \pm 5.38$ & $20.75 \pm 3.97$ & $1.471(1.308-1.655)$ & $<0.001$ & & \\
\hline CLIF-C ACLF & $41.96 \pm 9.76$ & $52.51 \pm 7.77$ & $38.74 \pm 7.83$ & $1.239(1.164-1.319)$ & $<0.001$ & & \\
\hline COSSH-ACLF & $6.21(5.49,7.04)$ & $7.96(6.91,8.92)$ & $5.86(5.31,6.49)$ & 7.237 (4.004-13.211) & $<0.001$ & & \\
\hline HINT & $-1.56(-2.50,-0.05)$ & $1.59(0,3.54)$ & $-2.10(-2.82,-1.04)$ & $2.80(2.07,3.79)$ & $<0.001$ & & \\
\hline P5 & $15.07(12.63,17.44)$ & $20.05(17.74,21.89)$ & $13.86(12.05,15.80)$ & $2.654(2.010-3.505)$ & $<0.001$ & & \\
\hline
\end{tabular}

UCIB, upper gastrointestinal bleeding; RR, relative risk; TSH, thyroid-stimulating hormone; CRP, C-reactive protein; ALT, alanine aminotransferase; AST, aspartate aminotransferase; HBsAg, HBV surface antigen.

In conclusion, we investigated the proteomes of patients with HBV-ACLF by TMT quantitative proteomics. The most promising biomarker, plasminogen, allowed the differentiation of HBV-ACLF nonsurvivors from survivors, whereas sequential plasminogen measurements facilitated predictions of the clinical course of HBV-ACLF.

We then showed that the P5 score was superior to currently available prognostic models and validated its performance in an 

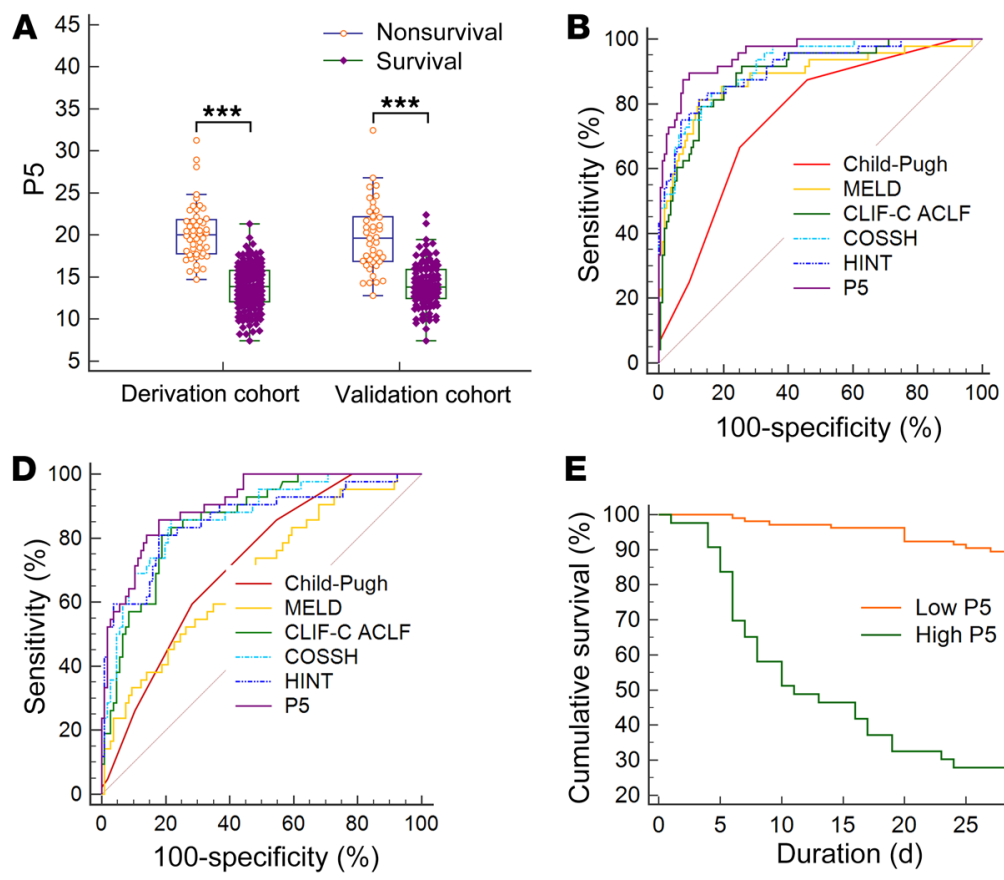

E

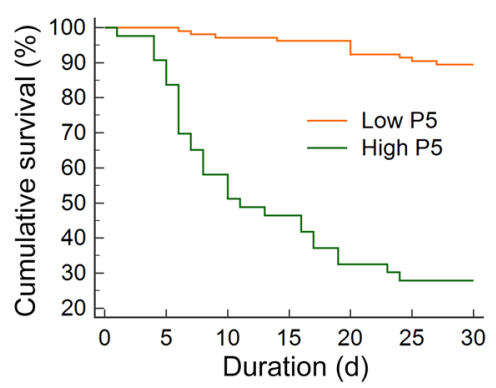

C

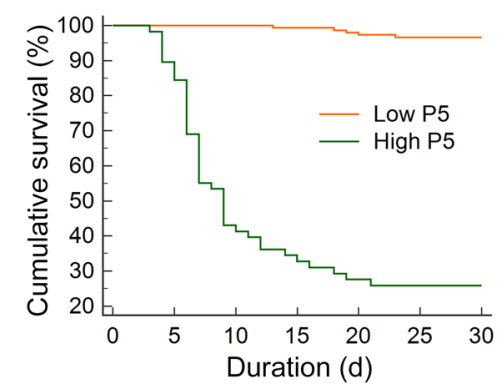

$\mathbf{F}$

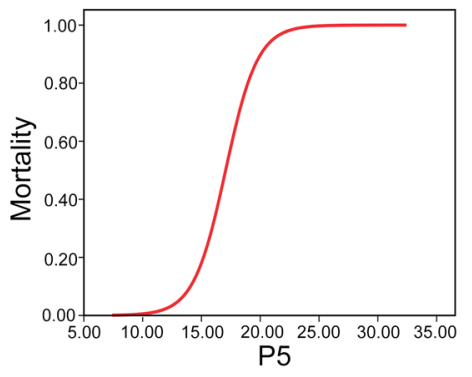

Figure 7. Correlations between the P5 score and HBV-ACLF prognosis. (A) P5 score distribution for patients with HBV-ACLF in the derivation and validation cohorts. ${ }^{* *} P<0.001$. Prediction of 30-day mortality for patients with HBV-ACLF according to the P5 and other scores in the (B) derivation and (D) validation cohorts. Thirty-day survival rates for the high-risk P5 (High P5) and low-risk P5 (Low P5) groups in the (C) derivation and (E) validation cohorts. Cumulative survival was compared by log-rank test $(P<0.0001$ for all). (F) Estimation of HBV-ACLF mortality based on the P5 score. Plasminogen levels were compared by the Mann-Whitney $U$ test $(\mathbf{A})$, Kaplan-Meier plots were compared by the log-rank test (C and $\mathbf{E})$, and the ROCs were compared by $z$ test (Delong's method) (B and D).

external, multicenter cohort. We believe that both the level of plasminogen and the P5 score, translated from bench to clinic, will facilitate clinical decision-making in the management of HBV-ACLF.

\section{Methods}

Definitions. CHB and LC were diagnosed according to criteria outlined in previous reports (33-36). HE was defined and graded using the West Haven criteria (37). HBV-AHD was diagnosed on the basis of previous studies (38-41). Briefly, patients with HBV-related CLD, with or without cirrhosis, experience acute hepatic insults resulting in liver dysfunction (manifesting as a serum TBil level $\geq 2 \mathrm{mg} / \mathrm{dL}$ and prolongation of the prothrombin time of $\geq 3$ seconds, with or without the presence of ascites, HE, or variceal hemorrhage) but do not meet the criteria for HBV-ACLF, and are thus considered to have HBV-AHD. HBV-ACLF was diagnosed according to slightly modified Asian Pacific Association for the Study of the Liver (APASL) criteria (30). In brief, HBV-ACLF was defined as TBil levels of $5 \mathrm{mg} / \mathrm{dL}$ or higher and an INR of 1.5 or higher (or prothrombin activity $<40 \%$ ) for patients with HBV-related CLD who have suffered acute hepatic insults within 4 weeks.

Organ failure was diagnosed using the chronic liver failuresequential organ failure assessment (CLIF-SOFA) score as described previously (2). Briefly, patients were considered to have liver failure if their TBil level was $12 \mathrm{mg} / \mathrm{dL}$ or higher and brain and kidney failure if they had grade III-IV HE and of a serum creatinine level of $2 \mathrm{mg} /$ $\mathrm{dL}$ or higher, respectively. Coagulation failure was confirmed when the INR was 2.5 greater or the platelet count was $20 \times 10^{9} / \mathrm{L}$ or lower. Circulation failure was determined when the mean arterial pressure was less than $70 \mathrm{mmHg}$ or when the patient was under treatment with vasoactive agents. Respiratory failure was determined when the ratio of arterial oxygen partial pressure to fractional inspired oxygen $\left(\mathrm{PaO}_{2} / \mathrm{FiO}_{2}\right)$ was 200 or less or the ratio of pulse oxymetric saturation to $\mathrm{FiO}_{2}\left(\mathrm{SpO}_{2} / \mathrm{FiO}_{2}\right)$ was 214 or less. The clinical course was assessed as described previously (30) by comparing the CLIF-SOFA scores at admission and on day 14 thereafter or at discharge. Improvement and deterioration were defined as a 2-point or greater decrease or increase in the CLIF-SOFA score, respectively, whereas fluctuation was defined as the difference between -1 and 1 .

Study design and patient enrollment. As shown in Figure 1, three batches of TMT-labeled quantitative proteomics analyses were performed for 10 patients with HBV-AHD and 20 patients with HBV-ACLF (10 survivors and 10 nonsurvivors). Then, 4 cohorts of subjects were enrolled for preliminary and further verification of screened biomarkers. A cross-sectional cohort (cohort 1), comprising $26 \mathrm{HCs}, 26 \mathrm{CHB}$, 26 HBV-related LC, 26 HBV-AHD, and 40 HBV-ACLF inpatients and outpatients from May 1 to August 1, 2016 from the First Affiliated Hospital of Zhejiang University, was enrolled for preliminary verification of those biomarker candidates identified by quantitative proteomics. A prospective, longitudinal HBV-ACLF cohort (cohort 2, derivation cohort, $n=207$ ), enrolled from June 1, 2017 to September 1, 2018 at the First Affiliated Hospital of Zhejiang University, was used to validate the prognostic performance of plasminogen and to develop a prognostic score for HBV-ACLF. We also included an external prospective, multicenter HBV-ACLF cohort (cohort 3, validation cohort, $n=148$ ), enrolled from June 1, 2016 to May 30, 2017 at the First Affiliated Hospital of Zhejiang University $(n=86)$, the Xiangya Hospital of Central South University $(n=35)$, and the Shulan Hospital of Hangzhou $(n=27)$. Cohort 3 
was used to further validate the performance of plasminogen and the P5 score. Finally, another cross-sectional cohort (cohort 4$)$, which included 30 HCs and 112 patients with autoimmune $(n=64)$ or alcoholic $(n=$ 48) CLD enrolled from July 1 to October 1, 2019 at the First Affiliated Hospital of Zhejiang University, was included to assess the performance of plasminogen in non-HBV-related ACLF. Among the 112 patients, 33 with presented noncirrhotic liver disease, 18 had LC, 33 had AHD, and 28 had ACLF. All patients with ACLF were followed for 30 days or until death, whichever occurred first. Exclusion criteria for patients with HBV-ACLF are detailed in the Supplemental Methods.

Management of patients with $\mathrm{HBV}$-ACLF. The management of patients with HBV-ACLF is detailed in the Supplemental Methods.

Proteomic protocols and bioinformatics analysis. High-abundance serum proteins (albumin and IgG) were depleted from samples using the ProteoPrep Blue Albumin and IgG Depletion Kit (PROTBA, Sigma-Aldrich). Next, $120 \mu \mathrm{g}$ albumin/IgG-depleted serum was reduced, alkylated, and digested with DTT, iodoacetamide (IAA), and trypsin. The peptide solution was labeled with a TMT-10plex Isobaric Label Reagent Set plus TMT11-131C Label Reagent (A34808, Thermo Fisher Scientific) and divided into 15 fractions. Fractions were dried, resuspended, and loaded onto a nanoACQUITY ultraperformance liquid chromatography (UPLC) system (Waters). Outcoming data were acquired using the Q-Exactive coupled mass spectrometer (Thermo Fisher Scientific). All protocols were performed according to the manufacturers' instructions (see also Supplemental Methods). RAW files were searched against the human UniProt database (version 11-2018; https://www.uniprot.org/ statistics/Swiss-Prot) using MaxQuant software (version 1.6.3.3). Bioinformatics analyses were performed using Perseus (version 1.6.1.3), Cytoscape (version 3.7.0), FunRich (version 3.1.3), and SIMCA (version 14.0) software, as described in the Supplemental Methods.

Western blotting, ELISA, and HNF-1 $\alpha$ interference in HepG2 cells. HNF-1 $\alpha$, a transcription factor predicted by FunRich software according to the DEPs, was validated by Western blotting on liver tissues from donors, recipients of liver transplantation, or liver biopsies. The levels of selected biomarker candidates in serum were measured using ELISA kits. The regulation of plasminogen by HNF-1 $\alpha$ was confirmed by detection of interference in HepG2 cells. Western blotting, ELISA, and HNF-1 $\alpha$ interference protocols are described in detail in the Supplemental Methods.

Calculation of scores. The Child-Pugh, MELD, CLIF-C ACLF, COSSH, and HINT scores were calculated as detailed in the Supplemental Methods.

Statistics. Statistical analysis was performed using SPSS (version 18.0, SPSS Inc.) and MedCalc (MedCalc Software). Categorical data, presented as numbers (percentages), were compared using $\chi^{2}$ or Fisher's exact test. Continuous data are presented in tables as the mean \pm SDs or the median with percentiles (P25-P75) and in graphs as dot plots with box-and-whisker plots. The lines within the boxes indicate the median, and the bounds of the boxes represent the 25th and 75th percentiles. Whiskers indicate both the maximum and minimum values, and dots out of whiskers represent outliers. Continuous data were compared by Student's $t$ test, 1-way ANOVA, Mann-Whitney $U$ test, or Kruskal-Wallis $\mathrm{H}$ test, as appropriate. Paired and repeated-measures data were analyzed by the Wilcoxon test and the Greenhouse-Geisser method, respectively. Rank correlation was analyzed by the Spearman method. Independent prognostic factors for HBV-ACLF were identified by multivariate logistic regression according to the forward Wald method, with entry and removal probabilities of 0.05 and 0.10 , respectively. Kaplan-Meier plots were used to compare the cumulative survival rates. A multivariate logistic regression analysis was performed to develop a prognostic score for HBV-ACLF. The receiver operating characteristic curves (ROCs) were compared using a $z$ test (DeLong's method). The HBV-ACLF mortality rate based on the P5 score was estimated by Fisher's canonical discriminant analysis. All tests were 2-tailed, and $P$ values of less than 0.05 were considered statistically significant.

Study approval. This study was conducted in compliance with the principles of the 1975 Declaration of Helsinki and was approved by the Ethics Committees of the First Affiliated Hospital, Zhejiang University, Xiangya Hospital, Central South University, and Shulan Hospital of Hangzhou. Written informed consent was obtained from all patients or their legal representatives prior to their participation in the study.

\section{Author contributions}

DW, SZ, and ZX contributed equally to this work. LL, ZS, and HG are the guarantors of this work. LL, HG, XL, and DZ designed the study. LL, ZS, HG, and XL obtained financial support for this study. DW, ZS, QR, and SZ conducted the main experiments. DW, SZ, EC, $\mathrm{QR}, \mathrm{KH}, \mathrm{ZX}, \mathrm{JY}$, and LX contributed to data acquisition. DW, QR, JY, LX, XD, NZ, BL, ZH, and BD collected serum and completed the follow-up. DW, KH, FJ, ZJ, YZ, and XO performed the serological detection tests. ZS, FJ, and DW conducted statistical analyses. DW, ZS, SZ, and XL analyzed and interpreted the data. DW wrote the manuscript, and ZS, HG, and LL provided critical revisions of the manuscript. All authors approved the final version of the manuscript.

\section{Acknowledgments}

This project was supported by grants from the National Key Research and Development Program (2017YFC1200204); the National Natural Science Foundation of China (81400589, 81600497); the Foundation for Innovative Research Groups of the National Natural Science Foundation of China (81121002); the Chinese High-Tech Research and Development Programs (2012AA020204; the National S\&T Major Project (2012ZX10002004); and the Zhejiang Provincial Medicine and Health Science and Technology Project (2016147735).

Address correspondence to: Zeyu Sun or Lanjuan Li, First Affiliated Hospital, College of Medicine, Zhejiang University, 79 Qingchun Road, Shangcheng District, 310003 Hangzhou, China. Phone: 86.571.87236759; Email: zeyusun@zju.edu.cn (ZS). Phone: 86.571.87236458; Email: ljli@zju.edu.cn (LL).
1. Bernal W, Jalan R, Quaglia A, Simpson K, Wendon J, Burroughs A. Acute-on-chronic liver failure. Lancet. 2015;386(10003):1576-1587.

2. Moreau R, et al. Acute-on-chronic liver failure is a distinct syndrome that develops in patients with acute decompensation of cirrhosis. Gastroenterology. 2013;144(7):1426-1437.e1.

3. Jalan R, et al. Acute-on chronic liver failure.

J Hepatol. 2012;57(6):1336-1348.

4. Trépo C, Chan HL, Lok A. Hepatitis B virus infec- tion. Lancet. 2014;384(9959):2053-2063.

5. Zheng $\mathrm{MH}$, et al. A model to determine 3-month mortality risk in patients with acute-on-chronic hepatitis B liver failure. Clin Gastroenterol Hepatol. 2011;9(4):351-356.e3. 
6. Sarin SK, et al. Acute-on-chronic liver failure: consensus recommendations of the Asian Pacific Association for the study of the liver (APASL). Hepatol Int. 2009;3(1):269-282.

7. Gustot T, et al. Clinical Course of acute-onchronic liver failure syndrome and effects on prognosis. Hepatology. 2015;62(1):243-252.

8. Olson JC, et al. Intensive care of the patient with cirrhosis. Hepatology. 2011;54(5):1864-1872.

9. Jalan R, Stadlbauer V, Sen S, Cheshire L, Chang YM, Mookerjee RP. Role of predisposition, injury, response and organ failure in the prognosis of patients with acute-on-chronic liver failure: a prospective cohort study. Crit Care. 2012;16(6):R227.

10. Jalan R, et al. The CLIF Consortium Acute Decompensation score (CLIF-C ADs) for prognosis of hospitalised cirrhotic patients without acute-on-chronic liver failure. J Hepatol. 2015;62(4):831-840.

11. Bachofner M, et al. Large-scale quantitative proteomics identifies the ubiquitin ligase Nedd4-1 as an essential regulator of liver regeneration. Dev Cell. 2017;42(6):616-625.e8.

12. Rowe $\mathrm{C}$, et al. Proteome-wide analyses of human hepatocytes during differentiation and dedifferentiation. Hepatology. 2013;58(2):799-809.

13. Wang J, et al. Proteomic signature of acute liver failure: from discovery and verification in a pig model to confirmation in humans. Mol Cell Proteomics. 2017;16(7):1188-1199.

14. Lv S, Wei L, Wang JH, Wang JY, Liu F. Identification of novel molecular candidates for acute liver failure in plasma of BALB/c murine model. JProteome Res. 2007;6(7):2746-2752.

15. Zhou N, et al. Discovery of a potential plasma protein biomarker panel for acute-on-chronic liver failure induced by hepatitis B virus. Front Physiol. 2017;8:1009.

16. Peng L, et al. iTRAQ-based proteomic analysis of hepatic tissues from patients with hepatitis B virus-induced acute-on-chronic liver failure. Exp Ther Med. 2015;10(5):1732-1742.

17. Ren F, et al. Comparative serum proteomic analysis of patients with acute-on-chronic liver failure: alpha-1-acid glycoprotein maybe a candidate marker for prognosis of hepatitis B virus infection. JViral Hepat. 2010;17(11):816-824.

18. von Wnuck Lipinski K, et al. Hepatocyte nuclear factor 1A deficiency causes hemolytic anemia in mice by altering erythrocyte sphingolipid homeostasis. Blood. 2017;130(25):2786-2798.

19. Pontoglio M, Pausa M, Doyen A, Viollet B, Yaniv M, Tedesco F. Hepatocyte nuclear factor 1alpha controls the expression of terminal complement genes. J Exp Med. 2001;194(11):1683-1689.

20. Babaya N, et al. Association of I27L polymorphism of hepatocyte nuclear factor-1 alpha gene with high-density lipoprotein cholesterol level. JClin Endocrinol Metab. 2003;88(6):2548-2551.

21. Garand M, Bastajian N, Nesheim ME, Boffa MB, Koschinsky ML. Molecular analysis of the human thrombin-activatable fibrinolysis inhibitor gene promoter. Br J Haematol. 2007;138(2):231-244.

22. Meroni G, Buraggi G, Mantovani R, Taramelli R. Motifs resembling hepatocyte nuclear factor 1 and activator protein 3 mediate the tissue specificity of the human plasminogen gene. Eur J Biochem. 1996;236(2):373-382.

23. Jalan $\mathrm{R}$, et al. Toward an improved definition of acute-on-chronic liver failure. Gastroenterology. 2014;147(1):4-10.

24. Grønbæk H, et al. Macrophage activation markers predict mortality in patients with liver cirrhosis without or with acute-on-chronic liver failure (ACLF). JHepatol. 2016;64(4):813-822.

25. Xin J, et al. Serum macrophage inflammatory protein $3 \alpha$ levels predict the severity of HBV-related acute-on-chronic liver failure. Gut. 2016;65(2):355-357.

26. Balasubramaniyan $V$, et al. Importance of Connexin- 43 based gap junction in cirrhosis and acute-on-chronic liver failure. J Hepatol. 2013;58(6):1194-1200.

27. Okada K, Ueshima S, Imano M, Kataoka K, Matsuo $\mathrm{O}$. The regulation of liver regeneration by the plasmin/alpha 2-antiplasmin system. J Hepatol. 2004;40(1):110-116.

28. Shimizu M, et al. Mechanism of retarded liver regeneration in plasminogen activator-deficient mice: impaired activation of hepatocyte growth factor after Fas-mediated massive hepatic apoptosis. Hepatology. 2001;33(3):569-576.

29. Bezerra JA, et al. Plasminogen deficiency leads to impaired remodeling after a toxic injury to the liver. Proc Natl Acad Sci USA. 1999;96(26):15143-15148.

30. Wu D, et al. HINT: a novel prognostic model for patients with hepatitis B virus-related acute-on- chronic liver failure. Aliment Pharmacol Ther. 2018;48(7):750-760.

31. Wu T, et al. Development of diagnostic criteria and a prognostic score for hepatitis B virusrelated acute-on-chronic liver failure. Gut. 2018;67(12):2181-2191.

32. Li H, et al. Characteristics, diagnosis and prognosis of acute-on-chronic liver failure in cirrhosis associated to hepatitis B. Sci Rep. 2016;6:25487.

33. Sarri G, Westby M, Bermingham S, Hill-Cawthorne G, Thomas H, Guideline Development Group. Diagnosis and management of chronic hepatitis B in children, young people, and adults: summary of NICE guidance. $B M J$. 2013;346:f3893.

34. Terrault NA, et al. AASLD guidelines for treatment of chronic hepatitis B. Hepatology. 2016;63(1):261-283.

35. Sarin SK, et al. Asian-Pacific clinical practice guidelines on the management of hepatitis B: a 2015 update. Hepatol Int. 2016;10(1):1-98.

36. Bajaj JS, et al. Second infections independently increase mortality in hospitalized patients with cirrhosis: the North American Consortium for the Study of End-stage Liver Disease (NACSELD) experience. Hepatology. 2012;56(6):2328-2335.

37. Ferenci P, Lockwood A, Mullen K, Tarter R, Weissenborn K, Blei AT. Hepatic encephalopathy--definition, nomenclature, diagnosis, and quantification: final report of the working party at the 11th World Congresses of Gastroenterology, Vienna, 1998. Hepatology. 2002;35(3):716-721.

38. Chen $\mathrm{CH}$, et al. Entecavir vs. lamivudine in chronic hepatitis B patients with severe acute exacerbation and hepatic decompensation. J Hepatol. 2014;60(6):1127-1134.

39. Chien RN, Lin CH, Liaw YF. The effect of lamivudine therapy in hepatic decompensation during acute exacerbation of chronic hepatitis B. J Hepatol. 2003;38(3):322-327.

40. Jeng WJ, Sheen IS, Liaw YF. Hepatitis B virus DNA level predicts hepatic decompensation in patients with acute exacerbation of chronic hepatitis B. Clin Gastroenterol Hepatol. 2010;8(6):541-545.

41. Tsai WL, et al. Early entecavir treatment for chronic hepatitis B with severe acute exacerbation. Antimicrob Agents Chemother. 2014;58(4):1918-1921. 